\title{
A BERNSTEIN PROBLEM IN WARPED PRODUCTS
}

\author{
Juan A. Aledo and Rafael M. Rubio \\ Universidad de Castilla-La Mancha, Departamento de Matemáticas, E.S.I. Informática \\ 02071 Albacete, Spain; juanangel.aledo@uclm.es \\ Universidad de Córdoba, Departamento de Matemáticas \\ Campus de Rabanales, 14071 Córdoba, Spain; rmrubio@uco.es
}

\begin{abstract}
Uniqueness and non-existence of entire solutions to the minimal surface equation in warped products $\mathbf{R}^{2} \times{ }_{f} \mathbf{R}$ are provided. As a consequence of our results, the classical Bernstein's Theorem is extended.
\end{abstract}

\section{Introduction} form

In this paper we deal with the following nonlinear elliptic equation in divergence

$$
\operatorname{div}\left(\frac{D u}{f(u) \sqrt{f(u)^{2}+|D u|^{2}}}\right)=\frac{f^{\prime}(u)}{\sqrt{f(u)^{2}+|D u|^{2}}}\left\{2-\frac{|D u|^{2}}{f(u)^{2}}\right\}
$$

where $f: \mathbf{R} \rightarrow(0,+\infty)$ is a positive smooth function and the unknown function $u$ takes values on $\mathbf{R}^{2}, D$ and div being, respectively, the gradient and the divergence operators in $\mathbf{R}^{2}$ endowed with its Euclidean standard metric $g$.

As is well-known, $u: \mathbf{R}^{2} \rightarrow \mathbf{R}$ satisfies (1) if it is extremal among functions under interior variation for the area integral

$$
\int_{\mathbf{R}^{2}} f(u) \sqrt{f(u)^{2}+|D u|^{2}}
$$

This variational problem naturally arises in Riemannian Geometry. In fact, consider the standard metric $d t^{2}$ on the real line and take $f$ a positive smooth function defined on $\mathbf{R}$. Then the product manifold $\mathbf{R} \times \mathbf{R}^{2}$ endowed with the Riemannian metric

$$
\bar{g}=\pi_{\mathbf{R}}^{*}\left(d t^{2}\right)+f\left(\pi_{\mathbf{R}}\right)^{2} \pi_{\mathbf{R}^{2}}^{*}(g),
$$

where $\pi_{\mathbf{R}}$ and $\pi_{\mathbf{R}^{2}}$ stand for the projections onto $\mathbf{R}$ and $\mathbf{R}^{2}$, respectively, is a Riemanian warped product which we will represent by $\bar{M}=\mathbf{R} \times{ }_{f} \mathbf{R}^{2}$ [6]. Warped products were introduced by Bishop and O'Neill in [3] to construct a large class of complete manifolds of negative curvature.

Given a domain $\Omega$ in $\mathbf{R}^{2}$ and $u \in C^{\infty}(\Omega)$, the induced metric on $\Omega$ via the graph $\Sigma_{u}=\{(u(p), p): p \in \Omega\}$ is given by

$$
g_{u}=d u^{2}+f(u)^{2} g
$$

Then, $u$ is a critical point of (2) if and only if the associated graph $\Sigma_{u}$ has zero mean curvature. Namely, (1) is the minimal surfaces equation on $\mathbf{R}^{2}$ for graphs in $\bar{M}$. When $f \equiv 1$, the equation (1) reduces to the well-known minimal surfaces equation 
in $\mathbf{R}^{3}$, and the celebrated classical Bernstein Theorem states that the only entire solutions $u \in \mathcal{C}^{\infty}\left(\mathbf{R}^{2}\right)$ to this equation are the affine planes.

Observe that from Bernstein's Theorem, the only entire bounded solution to the minimal surfaces equation on $\mathbf{R}^{2}$ are the constant functions. For the general equation (1), if there exists $t_{0} \in \mathbf{R}$ such that $f^{\prime}\left(t_{0}\right)=0$, then the constant function $u=t_{0}$ is an entire solution to (1). Thus, the following questions arise in a natural way:

When the constant functions $u=t_{0}$ with $f^{\prime}\left(t_{0}\right)=0$ are the unique entire solutions to Eq. (1)?

When is there no entire solution to Eq. (1)?

The main aim of this paper is to give several answers to these questions under suitable geometrical assumptions. In the light of Bernstein's Theorem, it seems natural to work under the hypothesis of boundedness of the solutions. Some other conditions regarding the function $f$ will be required, all of them naturally extending the setting of the original Bernstein Theorem.

\section{Preliminaries}

Let us consider the standard metrics $d t^{2}$ and $g$ on the real line $\mathbf{R}$ and $\mathbf{R}^{2}$, respectively, and take $f$ a positive smooth function defined on $\mathbf{R}$. We will denote by $\bar{M}=\mathbf{R} \times{ }_{f} \mathbf{R}^{2}$ the manifold $\mathbf{R}^{3}=\mathbf{R} \times \mathbf{R}^{2}$ endowed with the Riemannian metric (3). We will denote by $T=\bar{\nabla} t$ the gradient of $t:=\pi_{\mathbf{R}}$ for the metric $\bar{g}$.

Let $\psi: S \rightarrow \bar{M}$ be an isometric immersion of a surface $S$ in $\bar{M}$. We will say that $S$ is two-sided if its normal bundle is trivial, i.e., we can choose a unit normal vector field $N$ globally defined on $S$ that we will call the Gauss map of the immersion $\psi$. Then we define the smooth non-oriented angle function $\theta: S \rightarrow[0, \pi)$ as

$$
\cos \theta(p)=\bar{g}(N(p), T(p)), \quad p \in S .
$$

Note that if $\psi$ is locally a graph on $\mathbf{R}^{2}$ (namely, $\psi$ is transversal to $T$ ), then it is two-sided and either $\cos \theta>0$ or $\cos \theta<0$ along $\psi$.

We will denote by $A$ the shape operator of $\psi$ with respect to $N$, and by $H=$ $\frac{1}{2} \operatorname{trace}(A)$ the mean curvature associated to $A$. Throughout this paper we will deal with minimal surfaces, i.e., surfaces with zero mean curvature. As is well-known, minimal surfaces are critical points of the area functional for compactly supported area variations.

The family of surfaces $S_{t}=\{t\} \times \mathbf{R}^{2}$ constitutes a foliation of $\bar{M}$ by complete totally umbilical leaves of constant mean curvature $(\log f)^{\prime}(t)=\frac{f^{\prime}(t)}{f(t)}$ that we will call slices. Note that the condition $(\log f)^{\prime \prime}(t) \geq 0\left(\operatorname{resp} .(\log f)^{\prime \prime}(t)>0\right)$ means that the mean curvature of the slices is non-decreasing (resp. strictly increasing). Moreover, note that these convexity conditions on the warping function are weaker than those used in [3].

We will say that a surface $\psi: S \rightarrow \bar{M}$ is contained in a slab if it is contained between two slices. In other words, the height function $\tau=\pi_{\mathbf{R}} \circ \psi$ of $\psi$ is such that $\tau(S) \subseteq\left[t_{1}, t_{2}\right]$ for $t_{1}, t_{2} \in \mathbf{R}, t_{1}<t_{2}$. Observe that a surface $\psi: S \rightarrow \bar{M}$ is contained in a slice if, and only if, $\cos ^{2} \theta=1$ along $\psi$.

\section{Set up}

Let $\psi: S \rightarrow \bar{M}$ be an isometric immersion of a surface $S$ in $\bar{M}=\mathbf{R} \times_{f} \mathbf{R}^{2}$. Next we will compute the Gaussian curvature $K$ of $S$. If we denote by $\overline{\operatorname{Ric}}$ and $\overline{\mathcal{S}}$ the 
Ricci curvature and the scalar curvature of $\bar{M}$, respectively, it is a straightforward computation (see [2, Section 2]) to check that

$$
K=\frac{1}{2} \overline{\mathcal{S}}-\overline{\operatorname{Ric}}(N, N)+2 H^{2}-\frac{1}{2} \operatorname{trace}\left(A^{2}\right) .
$$

On the other hand, we have that (see [6, Ex. 7.13])

$$
\overline{\mathcal{S}}=-4 \frac{f^{\prime \prime}}{f}-2 \frac{f^{\prime 2}}{f^{2}}
$$

Let us put $N^{M}=N-\bar{g}(N, T) T$. Then

$$
\overline{\operatorname{Ric}}(N, N)=\overline{\operatorname{Ric}}\left(N^{M}, N^{M}\right)+\cos ^{2} \theta \overline{\operatorname{Ric}}(T, T)
$$

can be computed using $[6$, Cor. 7.43$]$ to obtain

$$
\overline{\operatorname{Ric}}(N, N)=\sin ^{2} \theta(\log f)^{\prime \prime}(t)-2 \frac{f^{\prime \prime}(t)}{f(t)} .
$$
we get

Finally, from (4), (5), (6) and using also the Gauss and Codazzi equations of $\psi$,

Lemma 1. Let $\psi: S \rightarrow \bar{M}$ be a minimal surface $S$ in $\bar{M}=\mathbf{R} \times{ }_{f} \mathbf{R}^{2}$. Then the Gaussian curvature $K$ of $S$ can be expressed as

$$
K=-\frac{f^{\prime}(\tau)^{2}}{f(\tau)^{2}}-\sin ^{2} \theta(\log f)^{\prime \prime}(\tau)-\frac{1}{2} \operatorname{trace}\left(A^{2}\right) .
$$

On the other hand, the gradient of the height function $\tau$ and $f(\tau) \cos \theta$ on $S$ are given, respectively, by

$$
\nabla \tau=T^{\top}=T-\cos \theta N
$$

and

$$
\nabla(f(\tau) \cos \theta)=-f(\tau) A\left(T^{\top}\right),
$$

where by ()$^{\top}$ we mean taking the tangential component of a vector field along $\psi$. Then, for a minimal surface, the Laplacians of $\tau$ and $f(\tau) \cos \theta$ on $S$ become, respectively,

$$
\Delta \tau=\frac{f^{\prime}(\tau)}{f(\tau)}\left(2-\sin ^{2} \theta\right)
$$

and

$$
\Delta(f(\tau) \cos \theta)=-f(\tau) \cos \theta \sin ^{2} \theta(\log f)^{\prime \prime}(\tau)-f(\tau) \cos \theta \operatorname{trace}\left(A^{2}\right),
$$

see [1] for the details. We will also use that, for a minimal surface, we have

$$
|\nabla(f(\tau) \cos \theta)|^{2}=\frac{1}{2} \operatorname{trace}\left(A^{2}\right)\left(f(\tau)^{2}-f(\tau)^{2} \cos ^{2} \theta\right) .
$$

In fact, let us take a local orthonormal frame $\left\{E_{1}, E_{2}\right\}$ on $S$ with $A E_{1}=\mu E_{1}$ and $A\left(E_{2}\right)=-\mu E_{2}$ for a certain function $\mu$. If we put $T^{\top}=k_{1} E_{1}+k_{2} E_{2}$ for certain functions $k_{1}$ and $k_{2}$, then

$$
\bar{g}\left(A T^{\top}, A T^{\top}\right)=\mu^{2}\left(k_{1}^{2}+k_{2}^{2}\right)=\frac{1}{2} \operatorname{trace}\left(A^{2}\right)\left|T^{\top}\right|^{2}=\frac{1}{2} \operatorname{trace}\left(A^{2}\right) \sin ^{2} \theta
$$

which jointly (8) allows to obtain (11).

For each $u \in C^{\infty}\left(\mathbf{R}^{2}\right)$, let $\Sigma_{u}=\left\{(u(p), p): p \in \mathbf{R}^{2}\right\}$ be the entire graph defined by $u$ on $\mathbf{R}^{2}$. The subset $\Sigma_{u}$ can be seen as a regular surface in $\bar{M}$ which inherits from $\bar{M}$ a Riemannian metric which, on $\mathbf{R}^{2}$, is given by

$$
g_{u}=d u^{2}+f(u)^{2} g \text {. }
$$


Note that $\tau(u(p), p)=u(p)$ for any $p \in \mathbf{R}^{2}$, and so $\tau$ and $u$ may be naturally identified on $\Sigma_{u}$. Observe also that $\Sigma_{u}$ is two sided, and we can orient the graph in such a way that $\cos \theta>0$. More precisely, the Gauss map of $\Sigma_{u}$ is given by

$$
N=\frac{f(u)}{\sqrt{f(u)^{2}+|D u|^{2}}}\left(T-\frac{1}{f(u)^{2}} D u\right)
$$

where $D u$ denotes the gradient of $u$ in $\left(\mathbf{R}^{2}, g\right)$ and $|D u|^{2}=g(D u, D u)$, and so

$$
\cos \theta=\frac{f(u)}{\sqrt{f(u)^{2}+|D u|^{2}}} .
$$

The graph $\Sigma_{u}$ is minimal if and only if $u$ is a solution to the non-linear elliptic equation (1).

\section{Main results}

In order to obtain our results, we will consider on $\mathbf{R}^{2}$ a metric $\widehat{g}_{u}$, conformal to $g_{u}$, which lets a certain control of its Gaussian curvature.

Lemma 2. Let $u \in C^{\infty}\left(\mathbf{R}^{2}\right)$ be a smooth function on $\mathbf{R}^{2}$ such that $\Sigma_{u}$ is a minimal graph in $\mathbf{R} \times_{f} \mathbf{R}^{2}$, where the smooth function $f: \mathbf{R} \rightarrow(0,+\infty)$ satisfies that $(\log f)^{\prime \prime} \geq 0$. Then

$$
(f(u) \cos \theta+\lambda)^{2} \widehat{K}_{u} \geq-\frac{f^{\prime}(u)^{2}}{f(u)^{2}}-(\log f)^{\prime \prime}(u) \sin ^{2} \theta\left(1+\frac{f(u) \cos \theta}{f(u) \cos \theta+\lambda}\right),
$$

where $\lambda=\inf f$ and $\widehat{K}_{u}$ denotes the Gaussian curvature of $\mathbf{R}^{2}$ furnished with the conformal metric

$$
\widehat{g}_{u}=(f(u) \cos \theta+\lambda)^{2} g_{u} .
$$

Proof. Using (10) and (11), if we denote by $\Delta_{u}$ the Laplacian operator on $\left(\mathbf{R}^{2}, g_{u}\right)$ we get

$\Delta_{u} \log (f(u) \cos \theta+\lambda)=\frac{1}{(f(u) \cos \theta+\lambda)^{2}}\left\{-\frac{1}{2} \operatorname{trace}\left(A^{2}\right) f(u)^{2} \sin ^{2} \theta\right.$

$$
\left.-f(u) \cos \theta(f(u) \cos \theta+\lambda)\left(\operatorname{trace}\left(A^{2}\right)+\sin ^{2} \theta(\log f)^{\prime \prime}(u)\right)\right\} .
$$

Since $f(u) \geq \lambda$, we have that

$$
f(u)^{2} \sin ^{2} \theta \geq \lambda^{2}-f(u)^{2} \cos ^{2} \theta
$$

and so

$$
\begin{aligned}
& \frac{1}{2} \operatorname{trace}\left(A^{2}\right) f(u)^{2} \sin ^{2} \theta+f(u) \cos \theta(f(u) \cos \theta+\lambda) \operatorname{trace}\left(A^{2}\right) \\
& \geq \frac{1}{2}(f(u) \cos \theta+\lambda)^{2} \operatorname{trace}\left(A^{2}\right) .
\end{aligned}
$$

Hence, from (15) it follows that

$$
\Delta_{u} \log (f(u) \cos \theta+\lambda) \leq \frac{f(u) \cos \theta}{f(u) \cos \theta+\lambda} \sin ^{2} \theta(\log f)^{\prime \prime}(u)-\frac{1}{2} \operatorname{trace}\left(A^{2}\right) .
$$

Now, consider on $M$ the metric $\widehat{g}_{u}$ conformal to $g_{u}$ given by (14). Then, from the well-known relation

$$
(f(u) \cos \theta+\lambda)^{2} \widehat{K}_{u}=K_{u}-\Delta_{u}(\log (f(u) \cos \theta+\lambda)),
$$

where $K_{u}$ is the Gaussian curvature of the metric $g_{u}$, we conclude the thesis by using Lemma 1, (10) and (16). 
Remark 3. Observe that $g_{u}$ is a complete metric on $\mathbf{R}^{2}$ since $\left(\mathbf{R}^{2}, g\right)$ is complete. If additionally inf $f>0$, then $\widehat{g}_{u}$ is also complete.

Lemma 4. Let $f: \mathbf{R} \rightarrow(0,+\infty)$ be a smooth function such that $(\log f)^{\prime \prime} \geq 0$ and inf $f>0$. Then, every bounded entire solution $u$ to Eq. (1) satisfies that $f^{\prime}(u)=0$.

Proof. Since we are assuming that $\Sigma_{u}$ is contained in a slab (because we are considering bounded solutions of (1)), we infer from Lemma 2 that $\widehat{K}_{u}$ is bounded from below.

We will use the following generalized maximum principle for Riemannian manifolds given by Omori [5] (see also Yau's paper [7]):

Let $M$ be a complete Riemannian manifold whose Ricci curvature is bounded away from $-\infty$ and let $u: M \rightarrow \mathbf{R}$ be a smooth function on $M$.

a) If $u$ is bounded from above on $M$, then for each $\varepsilon>0$ there exists a point $p_{\varepsilon} \in \mathbf{R}^{2}$ such that

$$
\left|\nabla u\left(p_{\varepsilon}\right)\right|<\varepsilon, \quad \Delta u\left(p_{\varepsilon}\right)<\varepsilon, \quad \sup u-\varepsilon<u\left(p_{\varepsilon}\right) \leq \sup u ;
$$

b) If $u$ is bounded from below on $M$, then for each $\varepsilon>0$ there exists a point $p_{\varepsilon} \in M$ such that

$$
\left|\nabla u\left(p_{\varepsilon}\right)\right|<\varepsilon, \quad \Delta u\left(p_{\varepsilon}\right)>-\varepsilon, \quad \inf u \leq u\left(p_{\varepsilon}\right)<\inf u+\varepsilon .
$$

Here $\nabla u$ and $\Delta u$ denote, respectively, the gradient and the Laplacian of $u$.

Thus, let $u \in C^{\infty}\left(\mathbf{R}^{2}\right)$ be a bounded solution to the differential elliptic equation (1) and consider $u$ as a smooth function on the complete Riemannian manifold $\left(\mathbf{R}^{2}, \widehat{g}_{u}\right)$ (see Remark 3 ). If we denote by $\widehat{\Delta}_{u}$ the laplacian operator on $\left(\mathbf{R}^{2}, \widehat{g}_{u}\right)$, then we have that

$$
\widehat{\Delta}_{u} u=\frac{1}{(\lambda+f(u) \cos \theta)^{2}} \Delta_{u} u
$$

As $u$ is bounded from below, from the generalized maximum principle we know that for each entire $m$ there exists a point $p_{m} \in M$ such that

$$
-\frac{1}{m}<\widehat{\Delta}_{u} u\left(p_{m}\right)
$$

Then, letting $m \rightarrow \infty$ and using (9) and (17), we obtain that $\frac{f^{\prime}(\inf u)}{f(\inf u)} \geq 0$. Reasoning analogously, since $u$ is bounded from above we obtain that $\frac{f^{\prime}(\sup u)}{f(\sup u)} \leq 0$. The thesis follows immediately by using that $(\log f)^{\prime \prime} \geq 0$.

Remark 5. Let $f: \mathbf{R} \rightarrow(0,+\infty)$ be a smooth positive function such that $(\log f)^{\prime \prime}>0$. Then it is clear that if there exists $t_{0} \in \mathbf{R}$ such that $f^{\prime}\left(t_{0}\right)=0$, then $t_{0}$ is the unique zero of $f^{\prime}$ and moreover inf $f>0$.

Now, let us relax the assumption $(\log f)^{\prime \prime}>0$ to $(\log f)^{\prime \prime} \geq 0$. Let us take $J=$ $\left\{t \in \mathbf{R}: f^{\prime}(t)=0\right\}$ and suppose that $J \neq \emptyset$ and it is not unitary. Then $(\log f)^{\prime \prime}(t)=0$ for every point $t \in J$. To see this, observe that the condition $(\log f)^{\prime \prime} \geq 0$ implies that $f^{\prime \prime} \geq 0$. Thus, if there exists $t_{0} \in J$ such that $(\log f)^{\prime \prime}\left(t_{0}\right)>0$, then it would be $f^{\prime \prime}\left(t_{0}\right)>0$ and so $f$ would attain a local minimum at $t_{0}$. Therefore $f^{\prime}$ would be strictly increasing in an open interval $\left(t_{0}-\epsilon, t_{0}+\epsilon\right)$ for a suitable real number $\epsilon>0$, i.e., $f^{\prime}$ would be negative on $\left(t_{0}-\epsilon, t_{0}\right)$ and positive on $\left(t_{0}, t_{0}+\epsilon\right)$. But then, since $f^{\prime}$ is non-decreasing on $\mathbf{R}$, no other zero of $f^{\prime}$ can exist.

Observe that, in general, provided that $(\log f)^{\prime \prime} \geq 0$ the existence of a zero of $f^{\prime}$ implies that inf $f>0$. 
As a consequence of Lemma 4, we obtain the following non-existence result

Theorem 6. Let $f: \mathbf{R} \rightarrow(0,+\infty)$ be a smooth function such that $(\log f)^{\prime \prime} \geq 0$ and $\inf f>0$. If the function $f^{\prime}$ has not zeros, then there is no bounded entire solutions to Eq. (1).

Also we have the following uniqueness result

Theorem 7. Let $f: \mathbf{R} \rightarrow(0,+\infty)$ be a smooth function satisfying that $(\log f)^{\prime \prime}>$ 0 . If there exists $u_{0} \in \mathbf{R}$ such that $f^{\prime}\left(u_{0}\right)=0$, then the only bounded entire solution to Eq. (1) is the constant function $u=u_{0}$.

Proof. The proof follows from Lemma 4 and Remark 5.

Remark 8. Note that if the function $f$ satisfies the weaker condition $(\log f)^{\prime \prime} \geq 0$ and there exists a unique zero of $f^{\prime}$, the thesis of Theorem 7 also holds.

Finally we get

Theorem 9. Let $f: \mathbf{R} \rightarrow(0,+\infty)$ be a smooth function satisfying that $(\log f)^{\prime \prime} \geq$ 0 . If there exists $u_{0} \in \mathbf{R}$ such that $f^{\prime}\left(u_{0}\right)=0$, then the only bounded entire solutions to Eq. (1) are the constant functions $u=c$ with $f^{\prime}(c)=0$.

Proof. We can suppose that the set $J$ is not unitary; otherwise, the result follows as has been reasoned above. Let $u$ be an entire bounded solution to Eq. (1). Using Lemma 2 and 4, and Remark 5, we have that

$$
(f(u) \cos \theta+\lambda)^{2} \widehat{K}_{u} \geq 0 .
$$

Consequently, $\widehat{K}_{u}$ is non-negative and $\left(\mathbf{R}^{2}, \widehat{g}_{u}\right)$ is parabolic (see [4, Sec. 4]).

Taking into account the invariance of harmonic functions by conformal changes of metric and using (9), we obtain that $u$ is harmonic and bounded on the parabolic surface $\left(\mathbf{R}^{2}, \widehat{g}_{u}\right)$. Therefore $u$ must be equal to a constant $c$, with $f^{\prime}(c)=0$.

Acknowledgments. The first author is partially supported by the Spanish MICINN Grant with FEDER funds MTM2013- 43970-P and by the Junta de Comunidades de Castilla-La Mancha Grant PEII-2014-001-A. The second author is partially supported by the Spanish MICINN Grant with FEDER funds MTM2013- 47828-C2-1-P.

\section{References}

[1] Alías, L. J., and M. DAJCzer: Constant mean curvature hypersurfaces in warped product spaces. - Proc. Edinb. Math. Soc. 50:3, 2007, 511-526.

[2] Alías, L. J., J. H. S. DE LiRA, and J. M. MalaCARne: Constant higher-order mean curvature hypersurfaces in Riemannian spaces. - J. Inst. Math. Jussieu 5:4, 2006, 527-562.

[3] Bishop, R. L., and B. O’Neill: Manifolds of negative curvature. - Trans. Amer. Math. Soc. 145:3, 1969, 1-49.

[4] Kazdan, J. L.: Parabolicity and the Liouville property on complete Riemannian manifolds. Aspects of Math. E10, ed. A. J. Tromba, Friedr. Vieweg and Sohn, Bonn, 1987, 153-166.

[5] Omori, H.: Isometric immersions of Riemannian manifolds. - J. Math. Soc. Japan 19, 1967, 205-214.

[6] O'Neill, B.: Semi-Riemannian geometry with applications to relativity. - Acad. Press, 1983.

[7] YAu, S. T.: Harmonic functions on complete Riemannian manifolds. - Commun. Pure Appl. Math. 28, 1975, 201-228. 